\title{
Estimación de dietas del chungungo Lontra felina (Molina, 1782) en dos localidades de la región del Biobío, Chile
}

\section{Estimation of the diet of chungungo Lontra felina (Molina, 1782) in two locations of the coast of the Biobío Region, Chile}

\author{
Aldo A. Poblete ${ }^{1 *}$, Konrad Górski ${ }^{1,2} \&$ Julio Moscoso ${ }^{3}$ \\ ${ }^{1}$ Departamento de Ecología, Facultad de Ciencias y Centro de Investigación en Biodiversidad y Ambientes Sustentables \\ (CIBAS), Universidad Católica de la Santísima Concepción, Av. Alonso de Ribera 2850, Concepción, Chile. \\ ${ }^{2}$ Instituto de Ciencias Marinas y Limnológicas, Facultad de Ciencias, Universidad Austral de Chile, Edificio Emilio Pugin, \\ Campus Isla Teja, Valdivia, Chile. \\ ${ }^{3}$ Ecogestion Ambiental Ltda. Consultora Ambiental. Progreso pje 1, № 1560 Chiguayante, Chile. \\ *aldopoblete.m@gmail.com
}

\begin{abstract}
RESUMEN
Chungungo Lontra felina (Molina, 1782) es un mustélido que presenta conducta exclusivamente marina con distribución desde Perú hasta Cabo de Hornos. Esta especie se encuentra categorizada En Peligro según UICN. Esto se debe principalmente a la caza ilegal, contaminación de las costas y explotación de sus recursos tróficos. De acuerdo a registros previos en distintas localidades de Chile, L. felina en sus hábitos alimenticios es una especie generalista, se alimenta principalmente de crustáceos, peces y moluscos según la oferta de cada localidad en que se encuentre. No obstante, no hay un estudio que determine y compare la alimentación de L. felina en localidades de la Región del Biobío. A través de métodos no invasivos, como el análisis fecal, es posible realizar una aproximación de la dieta, mediante presas no digeridas. De esta forma, con la finalidad de conocer la composición trófica del chungungo en la costa de la Región del Biobío se realizó un estudio de análisis fecal en dos localidades; Caleta Chome y San Vicente las cuales presentan características geográficas, antropogénicas y de contaminación distintas. En total se colectaron 131 restos fecales, 65 en Caleta Chome y 66 en San Vicente. En ambas localidades se encontró una baja diversidad de presas consumidas en comparación a estudios realizados en otras localidades de Chile, sin embargo, entre estas dos localidades se encontró una mayor diversidad de presas consumidas por L. felina en Caleta Chome que en San Vicente. La dieta de ambas localidades fue principalmente de crustáceos, seguido de peces y por último moluscos.
\end{abstract}

Palabras clave: alimentación, fecas, litoral Talcahuano, nutria marina, presas.

\begin{abstract}
Chungungo Lontra felina (Molina, 1782), is a marine mustelid. Its distribution extends from Peru to Cape Horn. This species is considered endangered throughout its entire range of distribution. The main threats to its populations are illegal hunting, coastal pollution and exploitation of their food resources. It has been reported that chungungo feeds mainly on crustaceans, fish and shellfish. Previous studies have compared feeding ecology of L. felina in some locations of Chile. There are, however, no reports that determine and compare feeding ecology of L. felina in the Biobío Region. Non-invasive methods such as fecal analyses allow to determine some properties of the diet by analyses of indigested prey or their parts. Present study was conducted in two locations in the Biobío Region: Caleta Chome and San Vicente and a total of 131 fecal remains was collected (65 in Caleta Chome and 66 in San Vicente). Fecal specimens were sieved and the remains of undigested prey were identified and categorised as crustaceans, fish or shellfish and counted. Furthermore, they were identified to the lowest possible taxonomic level using magnifying glass and identification keys available in literature. Low diversity of prey was found in both locations and the diet of chungungo consisted mainly of crustaceans, followed by fish and shellfish. Furthermore, greater variability and diversity of prey consumed by chungungo was found in Caleta Chome compared to San Vicente.
\end{abstract}

KEYwoRDS: faeces, feeding, marine otter, prey items, Talcahuano coast. 


\section{INTRODUCCIÓN}

Chungungo Lontra felina (Molina 1782) es un mamífero de la familia de los mustélidos, es el más pequeño y morfológicamente distintivo de su género taxonómico (Brownell 1978). A diferencia de las otras especies del género Lontra, que habitan en sistemas de agua dulce, el chungungo es el único que habita en ambientes marinos (Ostfeld et al. 1989). Su distribución se extiende desde Perú hasta Cabo de Hornos (Castilla \& Bahamondes 1979, Ebensperger \& Castilla 1992, Medina-Vogel et al. 2004) y su hábitat se limita principalmente al litoral rocoso expuesto al oleaje, específicamente cuevas formadas por rocas aunque también se le ha observado utilizando playas de arena. El área utilizada por L. felina abarca aproximadamente $4 \mathrm{~km}$ de costa, $100 \mathrm{~m}$ mar a dentro y $30 \mathrm{~m}$ hacia el interior continental (Castilla \& Bahamondes 1979, Cabello 1983, Sielfeld 1990, Ebensperger \& Castilla 1992, Medina-Vogel et al. 2004, 2008). L. felina es considerada como una especie principalmente solitaria, ocasionalmente observada integrando grupos de no más de tres individuos generalmente en épocas reproductivas (Housse 1953, Cabello 1983, Castilla 1981, Ostfeld et al. 1989).

De acuerdo a los antecedentes de reducción de población, la cual continuará disminuyendo dada la degradación acelerada del hábitat y ocupación humana, el Ministerio del Medio Ambiente la ha categorizado como especie Vulnerable A3c para todo su rango de distribución en Chile, lo que significa según el Reglamento de Clasificación de Especies (RCE) que L. felina perdería alrededor del $30 \%$ de su población actual en los próximos 30 años. Por otro lado, a nivel internacional la IUCN ha clasificado a $L$. felina como una especie En Peligro (Alvarez et. al. 2008, Valqui \& Rheingantz 2015), y está incluida dentro del Apéndice I de la Conservación sobre el Comercio Internacional de Especies Amenazadas de Fauna y Flora Silvestre (CITES, 2003). Durante el siglo XX hubo una drástica declinación de sus poblaciones y reducción de su distribución geográfica debido a la caza indiscriminada producto de la comercialización de su piel para la industria peletera (Iriarte \& Jacksic 1986). Esto ha originado poblaciones fragmentadas y aisladas a lo largo de su distribución lo cual podría ser un factor altamente negativo para la persistencia de la especie y mantención de sus poblaciones (MedinaVogel et. al. 2008). Actualmente, con la prohibición de las fábricas peleteras, las principales amenazas radican en la fuerte presión de diversas actividades antrópicas, donde se destaca la contaminación, urbanización, sobreexplotación de sus recursos tróficos y fragmentación del hábitat (Medina 1997, Siefeld \& Castilla 1999). Por esta razón, el estudio de los aspectos biológicos y ecológicos de cada una de sus poblaciones es fundamental para incorporar nueva información para desarrollar medidas de conservación y gestión eficiente en pro de la especie.
De acuerdo a Cursach et al. (2012), L. felina presenta una alta conducta sinantrópica al interactuar con humanos en ambientes con distintos grados de urbanización. Sin embargo, se considera a L. felina como una especie de conducta tímida y solitaria en general, exceptuando la época reproductiva, lo que hace difícil la observación directa de esta especie. Dada la complejidad de su observación directa es que existen métodos no invasivos para el estudio de esta especie, tales como avistamiento del uso de madrigueras, huellas (Badilla et. al. 2009) y análisis de muestras fecales (Villegas et al. 2007, Medina-Vogel et al. 2004, Ostfeld et al. 1989, Sielfeld 1990). Esta última utilizada especialmente para estudios de dieta, ya que correspondería al análisis más directo, confiable y sin daños a la población.

Se ha considerado a L. felina como un depredador de gran importancia en los ecosistemas costeros y con un alto nivel trófico (Castilla 1981, Soto 1996). Estudios previos acerca de la dieta y composición de presas de L. felina en diferentes localidades costeras, han determinado que se alimenta principalmente de peces, crustáceos y moluscos (Biffi \& Iannacone 2010, Córdova et al. 2009, Mangel et al. 2011, Medina- Vogel et al. 2008). Sin embargo, existen variaciones latitudinales en relación a la diversidad taxonómica de presas consumidas. Por ejemplo, para la zona costera de Valdivia se ha observado una mayor diversidad de presas; once especies de crustáceos donde la especie más consumida Talieptus dentatus, 9 especies de peces siendo la más consumida Eleginops maclovinus y 2 especies de moluscos, Octopus sp. y Fissurella sp. (Medina-Vogel et al. 2004). Para la localidad de Chiloé se han registrado 3 especies de crustáceos siendo Cancer setosus la presa más consumida, 3 especies de peces tales como Calliclinus genigultatus y Patagonothen sp. y 4 especies de moluscos siendo Collisella sp. es la presa más consumida (Ostfeld et al. 1989). En la zona norte, en la localidad de Pan de Azúcar se registró un total de 3 especies de crustáceos donde Rhynchocinetes typus es la presa más consumida, 5 especies de peces siendo Scartichthys viridis la presa más consumida y 3 especies de moluscos de los cuales Semimytilus algosus es la presa más consumida (Ostfield et. al. 1989) y en la localidad de Isla Choros se registró un total de 7 especies de crustáceos donde Petrolisthes sp. es la presa más consumida, 7 especies de peces siendo Girella laevifrons la presa más consumida y 3 especies de moluscos de los cuales Tegula atra es la presa más consumida (Villegas et. al. 2007).

Dado que existe una variación en la diversidad y composición de presas consumidas por L. felina en distintas localidades del país y el nulo conocimiento sobre sus hábitos tróficos en la Región del Biobío es altamente relevante un estudio que nos permita entender, mediante el análisis de muestras fecales, cómo es la composición en la dieta de $L$. felina en esta localidad y cómo contrastan los resultados obtenidos con los estudios ya realizados. 


\section{MÉTODOS}

Área de estudio

El estudio se realizó en dos localidades de la Región del Biobío (fig. 1), Caleta Chome ( $36^{\circ} 46^{\prime} 26^{\prime \prime} \mathrm{S}$ y $73^{\circ} 12^{\prime} 47$ O) y San Vicente $\left(36^{\circ} 43^{\prime} 42.5^{\prime \prime} \mathrm{S}, 73^{\circ} 08^{\prime} 13.2^{\prime \prime} \mathrm{O}\right)$, durante los meses de abril y mayo de 2016 .

Geográficamente Caleta Chome se ubica en el extremo sur de la península de Hualpén, y se caracteriza por ser una zona rocosa protegida, donde se desarrollan actividades como pesca artesanal, buceo deportivo y extracción de mariscos (Badilla et. al. 2009). Además, la Caleta se caracteriza por una baja intervención humana, transitada únicamente por habitantes de la zona. Por otro lado, la localidad de San Vicente se ubica en la zona norte de la Bahía San Vicente, presentándose como una bahía abierta y más expuesta. Presenta un alto grado de intervención humana debido al constante flujo de embarcaciones producto de la actividad portuaria que se realiza en el área.

COLECCIÓN DE MUESTRAS

Preliminarmente se realizaron entrevistas a pescadores y habitantes de ambas localidades, para obtener información relevante que permitiera agilizar las actividades tanto de avistamiento como colecta de muestras. La información

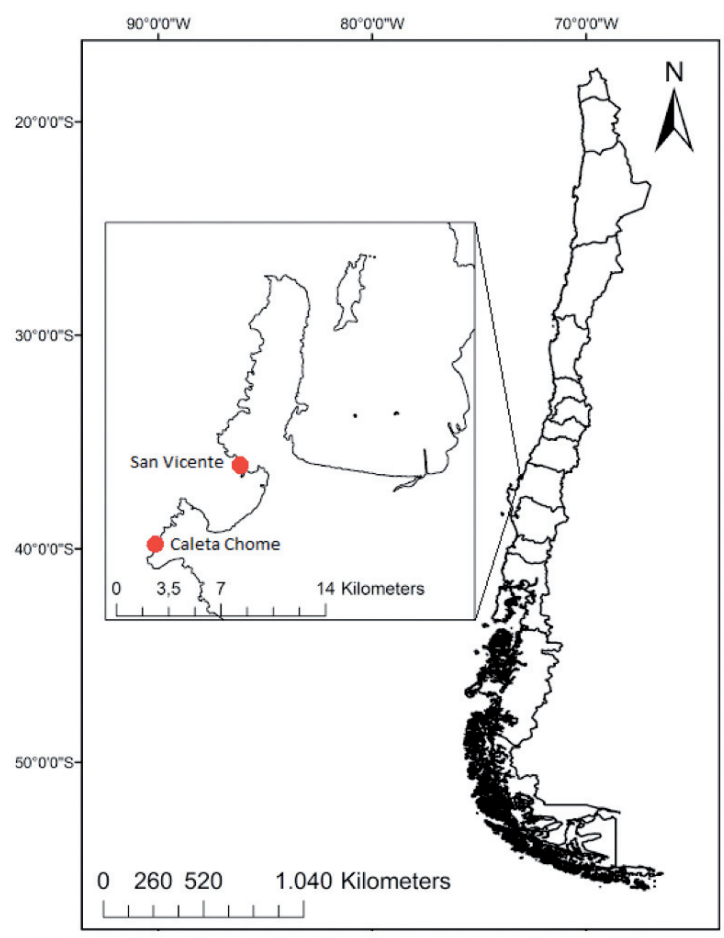

FiguRA 1. Localización específica del área de estudio en la zona costera del Talcahuano (recuadro) y su ubicación respecto a Chile latitudinal. / Specific study area in Talcahuano coastal zone and its location in Chile. específica que se obtuvo mediante estas entrevistas consistió en frecuencia de avistamiento, lugar del avistamiento, tiempo aproximado del último avistamiento y cantidad de individuos observados (Tabla 1).

En el estudio se utilizaron los restos de presas no digeridas presentes en las fecas de L. felina las cuales fueron colectadas en cuevas formadas entre los roqueríos alejados de lugares habitados (Fig. 2). Debido a la dificultad para determinar la procedencia de cada feca encontrada, las cuales podrían corresponder a uno o más individuos de L. felina o a uno o más eventos de excreción es que se consideró para este estudio todas aquellas fecas duras que se encontraban separadas entre sí presente en las cuevas.

Las muestras fecales fueron almacenadas en tubos falcon y preservadas en alcohol al $80 \%$, las cuales fueron transportadas al Laboratorio Biológico de Sedimentos de Ecogestion Ambiental Ltda. para su posterior análisis. Inicialmente cada feca se secó a temperatura ambiente, se obtuvo su peso mediante una balanza electrónica y fue tamizada en un tamiz de $300 \mu \mathrm{m}$, esto con el objetivo de evitar la pérdida de trozos no digeridos que pudieran ser útiles para la posterior identificación de presas. Una vez tamizado, el material obtenido fue secado a temperatura ambiente y pesado nuevamente, esto con el fin de determinar la cantidad relativa de material no digerido y el resto de material fecal que se perdió en tamizaje, el cual fue expresado en porcentaje.

Una vez finalizado el procedimiento anterior, se identificaron y agruparon las piezas duras de cada una de las presas las cuales podrían servir en la identificación de estas al menor nivel taxonómico posible. Para peces se agruparon escamas y otolitos, para crustáceos se agruparon trozos de caparazón, patas o tenazas y para moluscos se agruparon trozos de placas o valvas. Cada una de las piezas fue observada bajo estereoscopio (5x) y se tomó registro fotográfico para su mejor identificación el cual se llevó a cabo mediante el uso de claves dicotómicas, referencias bibliográficas y consultas a especialistas.

Adicionalmente se realizó un registro de aquellas piezas no digeridas que no calzaban con las tres categorías taxonómicas mencionadas anteriormente las cuales no corresponderían necesariamente a ítems alimentarios.

\section{EsTIMACIÓN DE ÍNDICES}

Para el análisis de presas no digeridas se utilizaron dos índices. Índice de frecuencia de aparición: $\mathrm{FA}=\mathrm{f} / \mathrm{N} * 100$, donde fi corresponde al número de fecas donde aparece la especie i y $\mathrm{N}$ corresponde al número total de fecas y el índice de proporción de aparición: $\mathrm{PA}=\mathrm{fi} / \mathrm{F} * 100$, donde $\mathrm{fi}$ corresponde al número de fecas en las que aparece la especie i y F corresponde al total de presas presentes en todas las fecas (Laroche 1982, Ruiz Olmo et al. 1989), esto con el fin de determinar cuál de las presas tiene mayor importancia en la alimentación de L. felina. 
TABLa 1. Preguntas que fueron utilizadas como información base para la identificación de sitios específicos de estudio en cada localidad. / Questions used to collect basic information in order to identify specific study sites in each locality.
1) ¿Ha visto nutrias en esta localidad?
2) ¿Hace cuánto tiempo tuvo el último avistamiento?
3) ¿Cuántos individuos pudo divisar?
4) ¿Cuántas nutrias cree que habitan en esta localidad?
5) ¿Puede identificar el sitio exacto donde ocurrió el avistamiento?

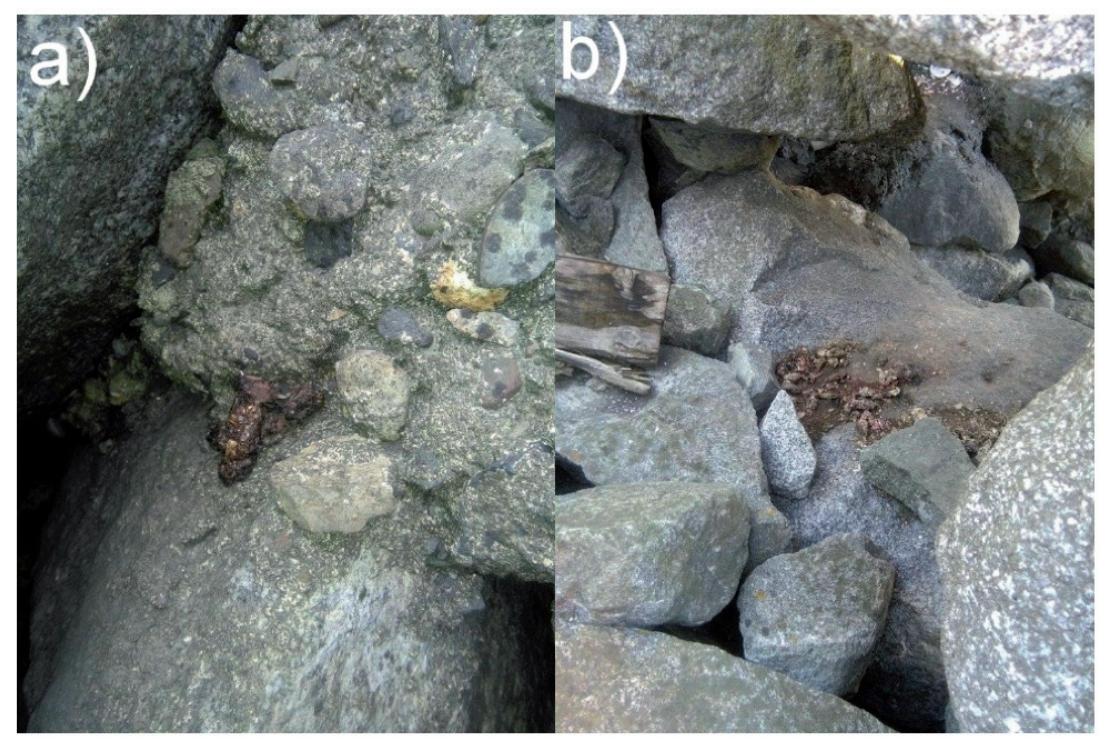

Figura 2. Imágenes de cuevas donde se encontraron muestras fecales (a) San Vicente, (b) Caleta Chome. / Images of caves where faecal samples were found (a) San Vicente, (b) Caleta Chome.

ANÁLISIS DE DATOS

Con el fin de estimar el porcentaje de material no digerido presente en cada feca, se calculó la proporción entre el peso de la muestra fecal en bruto y el peso una vez tamizado. Para evaluar si existen diferencias significativas en el peso de material no digerido presente en las fecas de cada localidad se realizó un análisis estadístico no paramétrico KruskalWallis.

Para determinar cuál de las dos localidades presenta una mayor diversidad de presas consumidas se utilizó el índice de Shannon-Weaver. Por último, para determinar la diferencia entre ambas localidades tanto en la composición como en la abundancia de presas consumidas por L. felina se realizó PERMANOVA de una vía (Anderson 2001).

\section{RESULTADOS}

Se analizó un total de 131 muestras fecales, 65 de Caleta Chome y 66 de San Vicente. Se determinó que del peso total de las muestras fecales colectadas en Caleta Chome, el 48,5 $\%$ aprox. corresponde a material no digerido, de igual manera se determinó que del peso total de las muestras fecales colectadas en San Vicente, el 59,7 \% aprox. corresponde a material no digerido, siendo el valor significativamente mayor para San Vicente $(\mathrm{H}=56,67, \mathrm{gl}=1, \mathrm{p}<0.05)$.

La frecuencia de aparición de las 65 fecas colectadas en Caleta Chome para el análisis de presas no digeridas registró para el ítem crustáceos un total de 91 piezas encontradas. Aproximadamente el $64 \%$ corresponde a presas del género Cancer, el $21 \%$ al género Talieptus, $8 \%$ a Pleurocondes monodon y el $4 \%$ Homalapsis plana (Tabla 2). Para el ítem molusco no se encontró ningún resto que podría corresponder a presa no digerida. En el ítem peces, no fue posible clasificar los restos encontrados a nivel de especie, sin embargo, se registraron 13 residuos de especies ícticas (Tabla 2).

Para San Vicente, la frecuencia de aparición de las 66 fecas colectadas, registró un total de 69 piezas, de las cuales el $84 \%$ corresponde a presas del género Cancer y el $15 \%$ 
a presas del género Talieptus (Tabla 2). El ítem molusco, de un total de tres piezas encontradas, se determinó que el $100 \%$ corresponde a presas del Semimytilus algosus (Tabla 2). Finalmente, para el grupo de los peces, los 19 restos de presas encontradas, no fue posible clasificar hasta el nivel de especie.

El análisis de diversidad estimado mediante el índice Shannon-Weaver para ambas localidades mostró bajos valores, con valores de H' para Caleta Chome de 1,2 y para San Vicente de 0,9. Se observó una diversidad más alta de las presas en Caleta Chome en comparación con San Vicente, sin embargo, esta diferencia no fue significativa.

$\mathrm{Se}$ encontraron diferencias significativas entre localidades en a la abundancia y composición de presas (PERMANOVA; $\mathrm{p}<0,01$ ). En ambas localidades analizadas, la dieta de L. felina fue compuesta principalmente de presas de crustáceos, seguida de peces. Los restos de moluscos fueron encontrados únicamente en las fecas de San Vicente (Fig. 5). Además, existe una mayor abundancia de presas crustáceos en Caleta Chome en comparación con San Vicente (Fig. 5). En los restos fecales analizados en este estudio, además de los restos del material alimentario, se identificaron también restos de materia que no corresponde necesariamente a alimento, como por ejemplo trozos de ramas, plásticos, plumas, pelos y entre otros restos que no fue posible categorizar. Estos restos fueron más frecuentes en San Vicente que en Caleta Chome.

\section{DISCUSIÓN}

El Análisis de la dieta de L. felina para las localidades de Caleta Chome y San Vicente ha permitido dilucidar la existencia de un alto consumo de presas de crustáceos en ambas localidades, y en menor proporción de peces y moluscos. Estos resultados son similares a los resultados obtenidos en estudios previos para otras localidades del norte y sur de Chile: Isla Chonos (Villegas et al. 2007), Valdivia (Medina-Vogel et al. 2004), Chiloé (Ostfeld et al. 1989) y Magallanes (Sielfeld 1990). Se observa diferencia con lo reportado para la localidad de Pan de Azúcar (Ostfeld et al. 1989) donde se estimó que la dieta para L. felina se basa principalmente en la ingesta de peces y en segundo lugar de crustáceos. En base a ello, se determinó que Caleta Chome y San Vicente presentan una menor diversidad de presas consumidas por L. felina en comparación a los estudios indicados anteriormente ya que la diversidad de presas no superó las cinco especies de crustáceos, y una especie de moluscos, mientras que, en otras localidades de Chile como Isla Choros, se ha reportado hasta siete especies de crustáceos, tres especies de moluscos y seis especies de peses en un total de 56 fecas analizadas (Villegas et al. 2007). Por otro lado, en Chiloé se han registrado solo tres especies de crustáceos y tres especies de peces, analizando un total de 24 fecas (Ostfield et al. 1989). En Crustáceos, esto se podría explicar debido a que se ha reportado un patrón de abundancia que disminuye hacia zonas más australes de Chile (Lancelotti \& Vásquez 2000), lo cual explicaría la diferencia en la presencia de los curstáceos.

TABLA 2. Número total y frecuencia relativa expresada en porcentaje (\%) para presas de crustáceos, moluscos y peces en muestras fecales de L. felina para cada localidad en estudio. / Total number and relative frequency of occurrence (\%) of crustaceans, shellfish and fish prey in fecal samples of L. felina in each studied zone.

\begin{tabular}{lcc}
\hline & Caleta Chome $(\mathrm{N}=65)$ & San Vicente $(\mathrm{N}=66)$ \\
\cline { 2 - 3 } Especies presa & Frecuencia de aparición de presas \\
\hline Crustáceos & $59(64,83 \%)$ & $58(84,05 \%)$ \\
Cancer setosus & $20(21,97 \%)$ & $11(15,94 \%)$ \\
Talieptus sp & $4(4,39 \%)$ & - \\
Homalapsis plana & $8(8,79 \%)$ & - \\
Pleurocondes monodon & 91 & 69 \\
Total & - & $3(100 \%)$ \\
Moluscos & & 3 \\
Semimytilus algosus & $13(100 \%)$ & $19(100 \%)$ \\
Total & 13 & 19 \\
\hline Peces & & \\
Sin Identificar & & \\
Total & & \\
\hline
\end{tabular}




\section{SAN VICENTE}
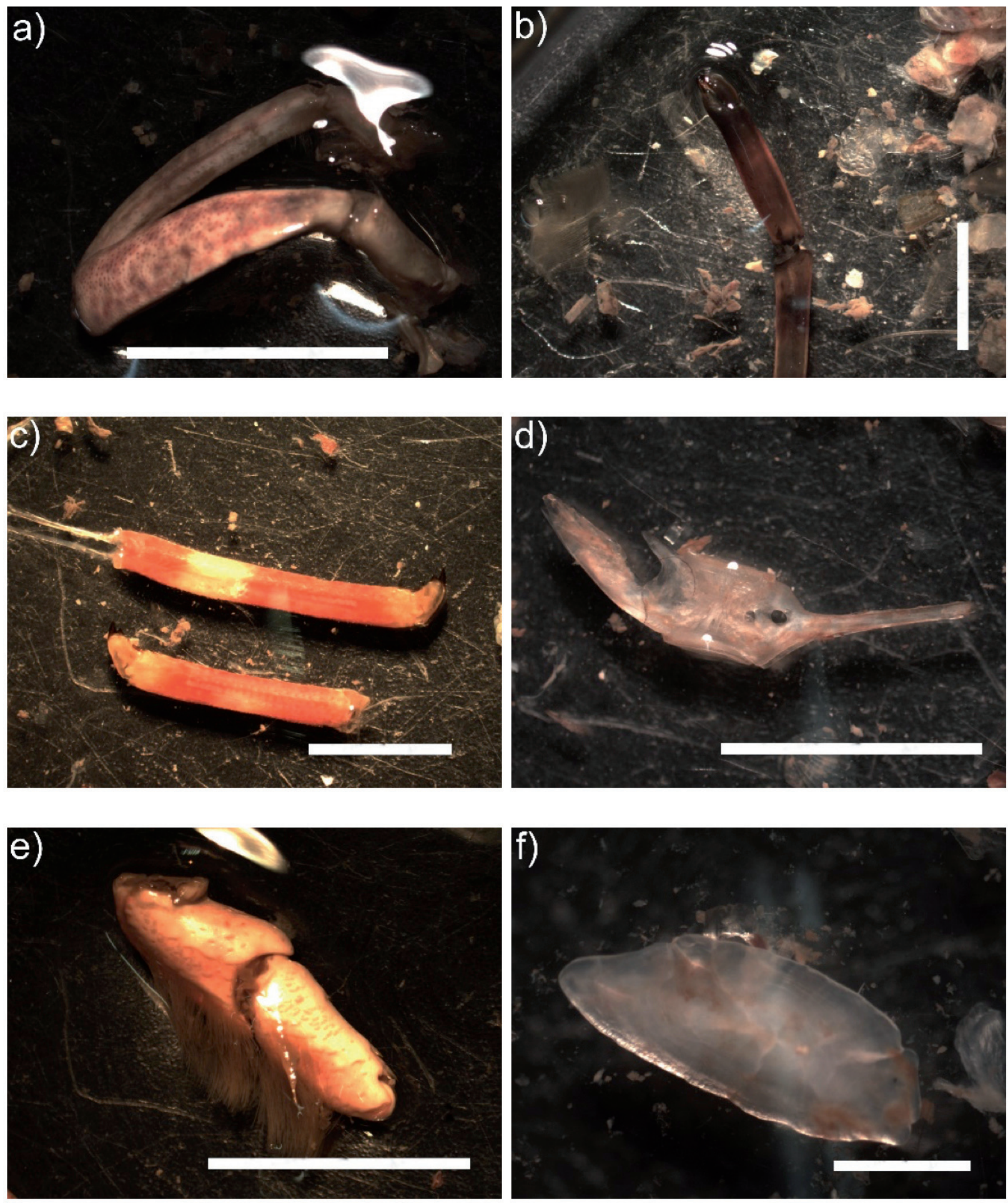

FIgURA 3. Imágenes de algunas presas encontradas en muestras fecales de Lontra felina en Caleta Chome. Barra de escala: a, b, c, d, e) $1 \mathrm{~cm}$ y f) $1 \mathrm{~mm}$. / Images of some prey items found in fecal samples of Lontra felina in Caleta Chome. Scale bar: a, b, c, d, e) $1 \mathrm{~cm}$ y f) $1 \mathrm{~mm}$. 


\section{CALETA CHOME}
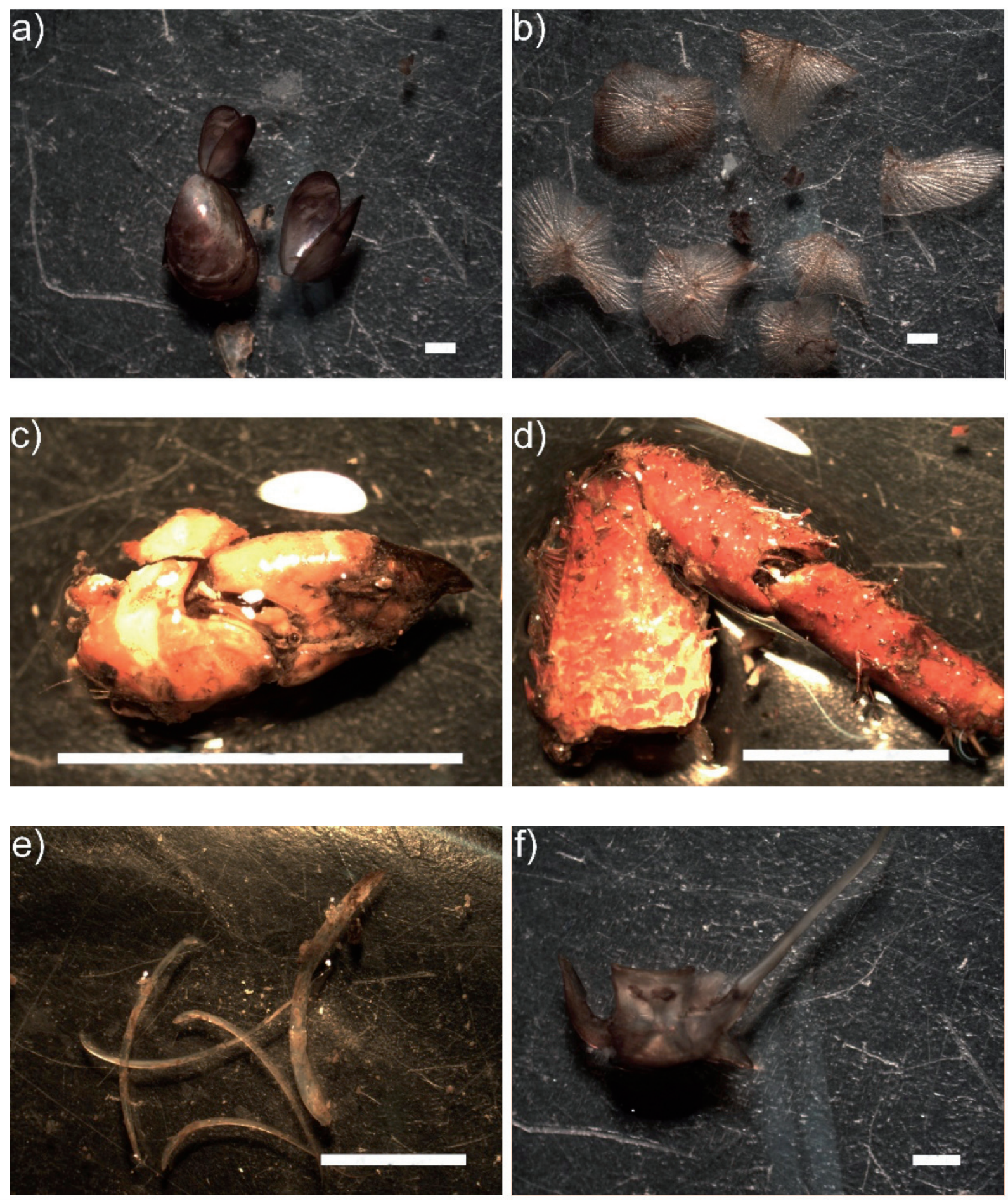

Figura 4. Imágenes de algunas presas encontradas en muestras fecales de Lontra felina en San Vicente. Barra de escala: c, d, e) $1 \mathrm{~cm}$ y a, b, f) $1 \mathrm{~mm}$. / Images of some prey items found in fecal samples of Lontra felina in San Vicente. Scale bar: c, d, e) $1 \mathrm{~cm}$ y a, b, f) $1 \mathrm{~mm}$. 


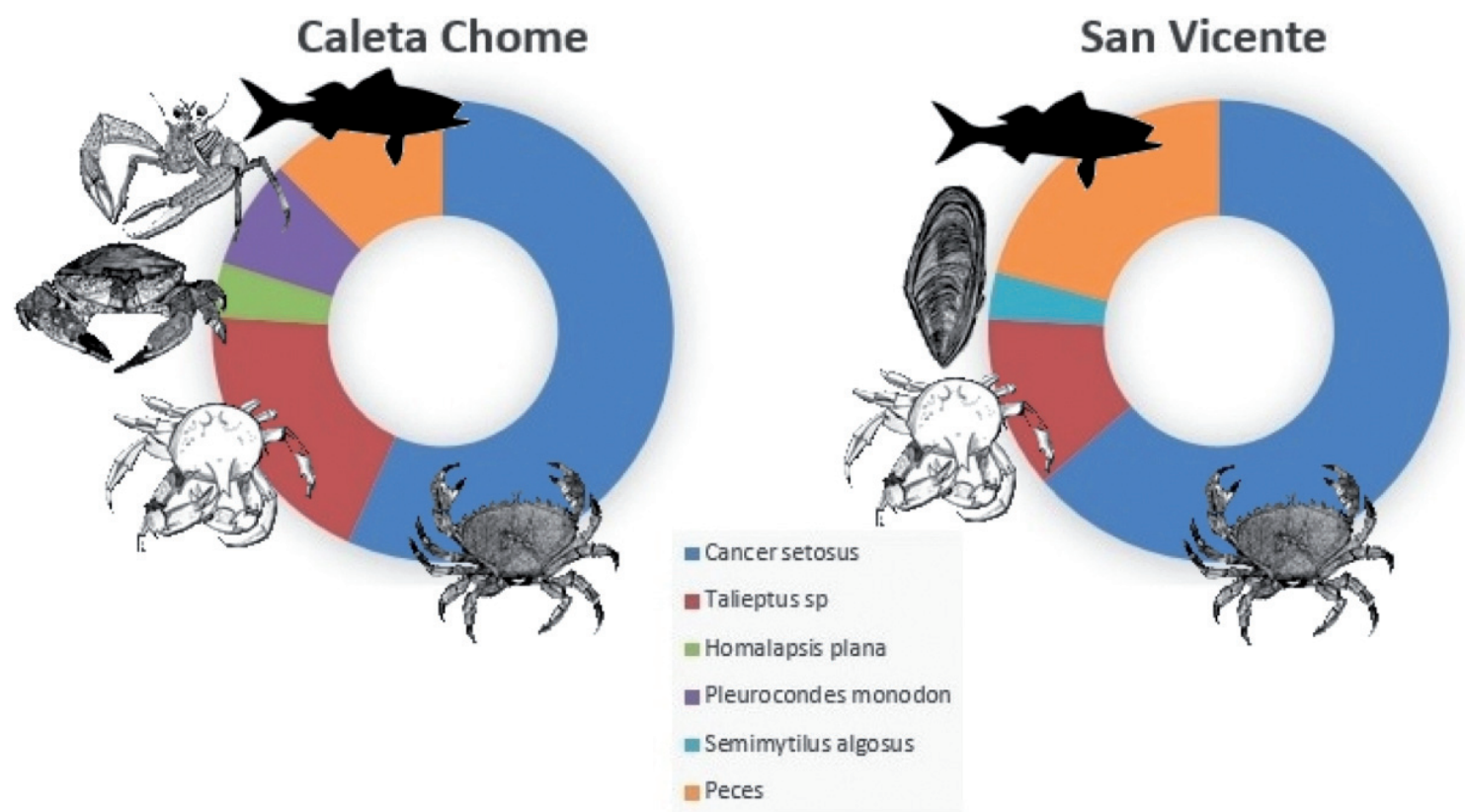

Figura 5. Abundancia relativa de presas en muestras fecales de L. felina en Caleta Chome y San Vicente. / Relative abundance of prey in fecal samples of L. felina in Caleta Chome and San Vicente.

La diferencia existente entre Caleta Chome y San Vicente, presentando la primera una mayor diversidad de presas consumidas, podría deberse a que ambas localidades presentan características geográficas y antropogénicas diferentes, lo cual podría ser un factor determinante en la presencia de alimento disponible para L. felina. La mayor presencia de material no correspondiente a alimento presente en las fecas de L. felina obtenidas en San Vicente podría ser explicado debido a la mayor influencia antrópica del lugar. Sin embargo, al no encontrar registro de este tipo de material en las fecas analizadas en otras localidades del país ni en estudios hechos con otras especies de mustélidos, se hace importante prestar atención a estos componentes ya que podrían indicar una alta presencia de material contaminante dentro de las áreas que ocupa L. felina el cual puede ser ingerido de forma accidental.

En ambas localidades se encontró una alta presencia de restos de peces como escamas o espinas en las muestras, dificultando su identificación debido a la baja o nula presencia de otolitos. Existen dos posibles explicaciones; L. felina no consume el pez completo, dejando la cabeza fuera de su alimentación, o bien, restos duros como otolitos permanecen por mayor tiempo en el tracto digestivo de L. felina (Biffi \& Iannacone 2010). Por otro lado, se ha registrado que $L$. felina consume peces que habitan en zonas tanto inter como sub mareal y con escasa movilidad (Sielfeld et al. 2006), razón por la que se considera necesario realizar un registro de ictiofauna presente en ambas localidades que permita conocer las potenciales presas de peces que pueda consumir L. felina para facilitar la identificación de presas consumidas por esta especie.

La información obtenida en este trabajo es una evidencia más para considerar L. felina como una especie generalista. La variedad de presas a lo largo de Chile sugiere que su dieta no se basa en una o más especies específicas, sino que depende de la disponibilidad de presas disponibles para su alimentación de acuerdo a la localidad donde se encuentre L. felina (Biffi \& Iannacone 2010, Córdova et al. 2009, Mangel et al. 2011, Medina-Vogel et al. 2004).

El estudio realizado proporciona información relevante sobre la alimentación de L. felina en la Región del Biobío, e incrementa el conocimiento existente sobre la especie y de esta población en particular, lo cual puede ser útil para futuras decisiones sobre estrategias de manejo y conservación. La variabilidad de presas consumidas por L. felina a lo largo de Chile puede estar condicionada principalmente por dos factores: las características geográficas y las especies de presas que habitan en cada localidad (Gerking 1994). El presente estudio está basado en solo dos localidades y numero de muestras limitado. Éste podría ser complementado con análisis que integre dieta en tiempo más prolongado, por ejemplo, análisis de isotopos estables en pelaje y presas disponibles y en un número mayor de localidades. 


\section{REFERENCIAS}

Anderson, M. 2001. A new method for non-parametric multivariate analysis of variance. Austral Ecology 26: 32-46

Alvarez, R., Medina-Vogel, G. 2008. Lontra felina. In: IUCN 2010. IUCN Red List of Threatened Species. Version 2010.4. URL: http://www.iucnredlist.org.

Badilla, M., George-Nascimento, M. 2009. Conducta diurna del chungungo L. felina (Molina 1782) en dos localidades de la costa de Talcahuano, Chile: ¿efectos de la exposición al oleaje y de las actividades humanas? Revista de Biología Marina y Oceanografía 44(2): 409-415.

Biffi, D., IANnACone, J. 2010. Variabilidad trófica de Lontra felina (Molina 1782) (Carnivora: Mustelidae) en dos poblaciones de Tacna (Perú) entre agosto y diciembre de 2006. Mastozoología Neotropical 17: 11-17.

BRownelL, R.L. JR. 1978. Ecology and conservation of the marine otter L. felina. In: Duplaix, N. (Ed.), Otters: Proceedings of the first working meeting of the otter specialist group: 104-106. International Union for Conservation of Nature and Natural Resources, Morges, Switzerland.

Cabello, C. 1983. La nutria de mar en la isla de Chiloé. Boletín Técnico, CONAF 6: 137.

Castilla, J.C. 1981 Perspectivas de investigación en estructura y dinámica de comunidades intermareales rocosas de Chile central. II Depredadores de alto nivel trófico. Medio Ambiente 5: 190-215.

CAstilla, J.C. 1982. Nuevas observaciones sobre conducta, ecología y densidad de Lontra felina (Molina 1782) (Carnivora: Mustelidae) en Chile. Publicación Ocasional, Museo Nacional de Historia Natural (Chile) 38: 197-206.

CASTILla, J.C., BAHAMONDES, I. 1979. Observaciones conductuales y ecológicas sobre Lontra felina (Molina) 1782 (Carnivora: Mustelidae) en las zonas central y centro-norte de Chile. Archivos de Biología y Medicina Experimentales 12: 119132.

Córdova, O., Rau, J., Suazo, C., Arriagada, A. 2009. Estudio comparativo de la ecología alimentaria del depredador de alto nivel trófico Lontra felina (Molina, 1782) (Carnivora: Mustelidae) en Chile. Revista de Biología Marina y Oceanografía 44: 429-438.

Cursach, J., Rau, J., Ther, F., Vilugrón, J., Tobar, C. 2012. Sinantroía y conservación marina: el caso del chungungo Lontra felina en el sur de Chile. Revista de Biología Marina y Oceanografía 47(4): 593-597.

Ebensperger, L.A., Castilla, J.C. 1992. Selección de hábitat en tierra por nutria marina, Lontra felina, en Isla Pan de Azúcar, Chile. Revista Chilena de Historia Natural 65: 429-434.

Gerking, S.D. 1994. Feeding ecology of fish. Academic Press, USA.

Helder, J., DE Andrade, K. 1997. Food and feeding habits of the neotropical river otter Lontra longicaudis (Carnivora, Mustelidae). Mammalia 61: 193-203.
Housse, R. 1953. Animales salvajes de Chile, en su clasificación moderna. Editorial Universidad de Chile, Santiago. 189 pp.

IrIARTE, J.A., JAKSIC, F.M. 1986. The fur trade in Chile: an overview of seventy-five years of export data (1910-1984). Biological Conservation 38: 243-253.

LAROCHE, J.L. 1982. Trophic patters among larvae of fish species of sculpins (Family: Cottidae) in marine stuary. Fishery Bulletin, Seattle (USA) 80: 827-840.

Mangel, J., Whitty, T., Medina-Vogel, G., Alfaro-Shigueto, J., CÁceres, C., Godley, B. 2011. Latitudinal variation in diet and patterns of human interaction in the marine otter. Marine Mammal Science 27: 14-25.

Medina-Vogel, G., Delgado, C.R., Alvarez, R.E., Bartheld, J.L.V. 2004. feeding ecology of the marine otter (Lontra felina) in a rocky seashore of the south of Chile. Marine Mammal Science 20: 134-144.

Medina-Vogel, G., Merino, L. O., Monsalve Alarcón, R., \& VIANNA, J. D. A. (2008). Coastal-marine discontinuities, critical patch size and isolation: implications for marine otter conservation. Animal Conservation 11(1): 57-64.

MedinA, G. 1997. A comparison of the diet and distribution of southern river otter (Lutra provocax) and mink (Mustela vison) in Southern Chile. Journal of Zoology 242: 291297.

Ostfeld, R.S., Ebensperger, L., Klosterman, L., Castilla, J.C. 1989. Foraging, activity budget, and social behavior of the South American marine otter Lontra felina (Molina, 1782). National Geographic Research 5: 422-438.

Ruiz Olmo, J., Jordán, G., Gosalbez, J. 1989. Alimentación de la nutria (Lutra lutra L., 1758) en el Nordeste de la Península Ibérica. Doñana Acta Vertebrata 16: 227-237.

Sielfeld, W. 1990. Características del hábitat de Lontra felina (Molina) y L. provocax Thomas (Carnivora, Mustelidae) en Fuego-Patagonia. Revista de Investigaciones Científicas y Tecnológicas, Serie Ciencias del Mar 1: 30-36.

Sielfeld, W., Castilla, J.C. 1999. Estado de conservación y conocimiento de las nutrias en Chile. Estudios Oceanológicos 18: 69-79.

Sielfeld, W., Guzmán, G., Amado, N. 2006. Distribución de peces del litoral rocoso de los canales patagónicos occidentales

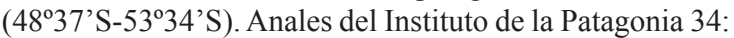
21-32.

Soто, R. 1996. Estructura gremial de un ensamble de depredadores de la zona intermareal rocosa en Chile central. Investigaciones Marinas 24: 97-105.

Valqui, J., Rheingantz, M.L. 2015. Lontra felina. Lista Roja de Especies Amenazadas de la UICN 2015: eT12303A21937779.

Villegas, M.J., Aron, A., Ebensperger, L.A. 2007. The influence of wave exposure on the foraging activity of marine otter, Lontra felina (Molina, 1782) (Carnivora: Mustelidae) in northern Chile. Journal of Ethology 25: 281-286.

Recibido: 04.06.2018

Aceptado: 04.11.2018 